\title{
Quadrados e polinômios do segundo grau com o uso do Algeplan
}

\author{
Karina Rodriguez de Fraga (D) Leandro Sebben Bellicanta (D)
}

\begin{abstract}
Resumo
Neste artigo é apresentada uma proposta de atividade didática utilizando o material concreto chamado Algeplan. Mais especificamente, o artigo explora a relação existente entre os coeficientes de alguns polinômios do segundo grau em duas variáveis e a quantidade de peças do Algeplan necessárias para se construir um quadrado. No desenvolvimento dessa atividade, os estudantes poderão descobrir uma maneira sistemática de contar e saber quais são os quadrados que podem ser construídos com as peças do Algeplan.
\end{abstract}

Palavras-chave: matemática, ensino, algeplan, material didático-pedagógico, álgebra.

\begin{abstract}
This paper presents a proposal for didactic activity using the concrete material called Algeplan. More specifically, the paper explores the relationship between the coefficients of some second-degree polynomials in two variables and the amount of Algeplan pieces needed to construct a square. In developing this activity students will be able to discover a systematic way to count and to know which squares can be constructed with Algeplan tiles.
\end{abstract}

Keywords: mathematics, teaching, algeplan, didactic-pedagogical material, algebra.

\section{Introdução}

Diversas pesquisas têm mostrado que o uso de materiais concretos no estudo de matemática tem um efeito potencializador no aprendizado e ajudam a estabelecer as bases para as fases subsequentes de abstração envolvidas no desenvolvimento do saber matemático do estudante [1].

Neste trabalho examina-se uma atividade prática, de fácil implementação, utilizando o bem conhecido material didático chamado Algeplan, que é constituído por peças retangulares de diferentes formatos e cores. O Algeplan usado neste trabalho está ilustrado na Figura 1 e possui um total 40 peças de madeira contendo:

- Quatro peças quadradas de cor amarela com lados medindo um certo valor que, neste trabalho, será denotado por $\mathrm{x}$.

- Quatro peças quadradas de cor azul com lados medindo um certo valor denotado por y. Observamos que os quadrados azuis possuem lado menor que os quadrados amarelos, de forma que $\mathrm{y}<\mathrm{x}$. 
- Doze quadrados de cor vermelha com lados medindo menos que y. Os lados desses quadrados serão tomados como a unidade de comprimento, ou seja, os lados serão denotados por 1.

- Oito peças retangulares de cor rosa de lados medindo y e 1.

- Oito peças retangulares de cor laranja de lados medindo x e 1.

- Quatro peças retangulares de cor verde de lados medindo x e y.

Figura 1: Peças do Algeplan

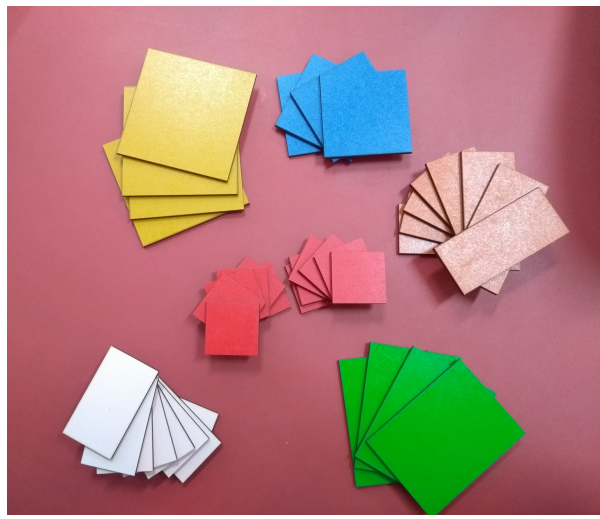

O Algeplan é um material particularmente adaptado ao estudo de polinômios do segundo grau pois, ao convencionarmos que a medida dos lados das peças são denotadas por x, y e 1, a área de cada uma dessas peças possui uma expressão algébrica que coincide com os monômios presentes em um polinômio de segundo grau nas variáveis x e y. Vários artigos e trabalhos acadêmicos exploram essa relação entre as peças do Algeplan e polinômios, conforme pode ser visto, por exemplo, em [2] e [3].

A Tabela 1 apresenta todas as peças do Algeplan com suas respectivas representações algébricas.

Tabela 1: Representações algébricas das peças do Alpeplan

\begin{tabular}{|c|c|c|c|}
\hline Quantidade de peças & Forma & Cor das peças & Representação algébrica \\
\hline \hline 4 & quadrado & amarelas & $\mathrm{x}^{2}$ \\
\hline \hline 4 & quadrado & azuis & $\mathrm{y}^{2}$ \\
\hline \hline 4 & retângulo & verdes & $\mathrm{xy}$ \\
\hline \hline 8 & retângulo & laranjas & $\mathrm{x}$ \\
\hline \hline 8 & retângulo & rosa & $\mathrm{y}$ \\
\hline 12 & quadrado & vermelhas & 1 \\
\hline
\end{tabular}

Dessa forma, pode-se associar a uma justaposição de peças do Algeplan uma expressão algébrica polinomial de grau dois, como mostram os seguintes exemplos:

Exemplo 1. O polinômio $y^{2}+x y+y+x+1$ pode ser representado no Algeplan justapondo-se uma peça azul $\left(y^{2}\right)$, uma peça verde (xy), uma peça rosa (y), uma peça laranja (x) e uma peça vermelha (1) que correspondem aos termos que estão sendo somados, conforme pode ser visualizado na Figura 2. 
Figura 2: Representação, com as peças do Algeplan, do polinômio $\mathrm{y}^{2}+\mathrm{xy}+\mathrm{y}+\mathrm{x}+1$ citado no exemplo 1

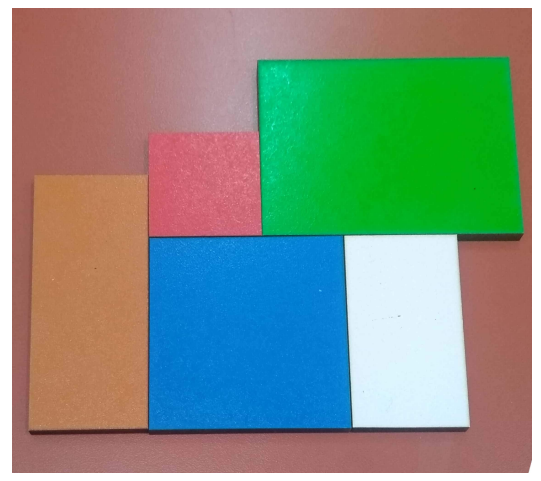

Exemplo 2. O polinômio $x^{2}+2 x y+y^{2}$ pode ser representado no Algeplan justapondo-se uma peça amarela $\left(\mathrm{x}^{2}\right)$, duas peças verdes $(\mathrm{xy})$ e uma peça azul $\left(\mathrm{y}^{2}\right)$, conforme pode ser visualizado na Figura 3 .

Figura 3: Representação, com as peças do Algeplan, do polinômio $x^{2}+2 x y+y^{2}$ citado no exemplo 2

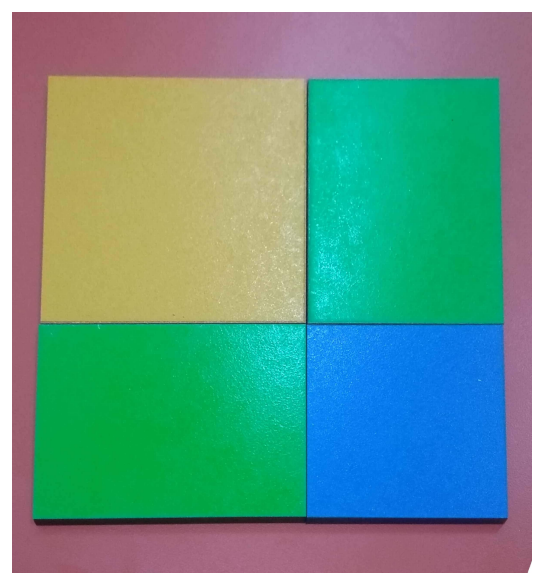

Tal associação entre a justaposição de peças do Algeplan com polinômios de grau dois permite-nos usar um material concreto no estudo dos polinômios, o que constitui um valioso recurso didático.

\section{O problema a ser abordado nesta atividade}

Ao manipularmos o Algeplan, algumas justaposições são especialmente atraentes pela regularidade alcançada na reunião das peças, como é o caso do quadrado que apareceu no exemplo 2.

Uma questão de enunciado fácil que pode despertar a curiosidade dos alunos que estejam usando o Algeplan em alguma atividade é a seguinte:

Questão 1. Quantos quadrados diferentes é possível montar usando as peças do Algeplan?

Para facilitar a resolução da questão 1, sugere-se que o significado da expressão “diferentes" seja interpretado como "não congruentes" de forma que os dois quadrados mostrados na Figura 4a serão contados apenas uma 
vez, pois são congruentes entre si, já que ambos possuem lados com medida y +1 . Dessa forma, os quadrados diferentes a que se refere a questão acima serão apenas os que possuem lados distintos, conforme os ilustrados na Figura 4b.

Figura 4: Quadrados usando o Algeplan.

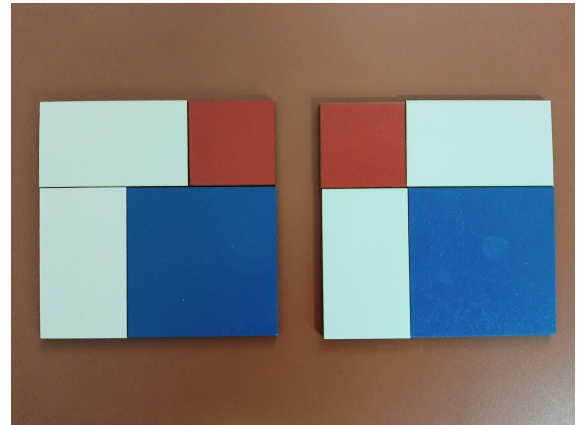

(a) Quadrados congruentes serão considerados iguais e contados apenas uma vez.

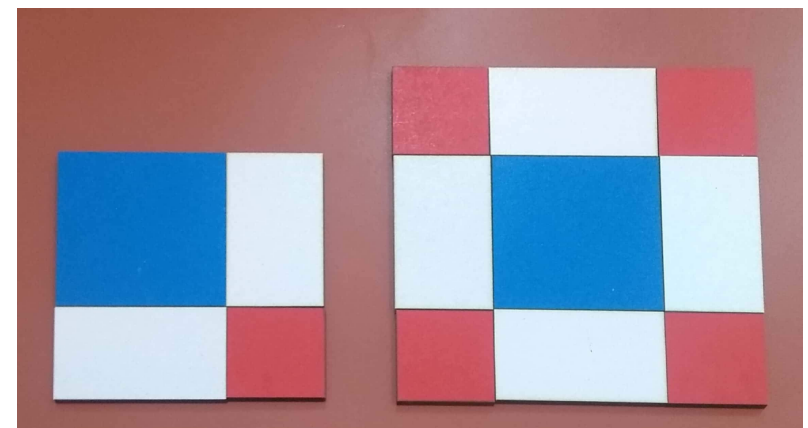

(b) Dois quadrados distintos montados com as peças do Algeplan.

\section{Quadrados geométricos e polinômios quadrados perfeitos.}

Para responder de forma definitiva à questão 1, pode-se usar da relação que existe entre as justaposições de peças do Algeplan com os polinômios de grau 2.

Afim de tornar mais clara e precisa essa relação entre peças do material concreto com os polinômios de grau dois, este trabalho usa os termos quadrados geométricos e polinômios quadrados perfeitos conforme as definições que seguem.

Definição 1 (Quadrado Geométrico). Um quadrado formado pela justaposição de uma ou mais peças do Algeplan será chamado de quadrado geométrico.

As figuras 4 e 5 mostram exemplos de quadrados geométricos.

Devido ao fato de as peças do Algeplan medirem x, y ou 1, o lado de um quadrado geométrico terá como medida possível $\alpha \mathrm{x}+\beta \mathrm{y}+\gamma$, onde $\alpha$ representa a quantidade de peças com lado $\mathrm{x}, \beta$ representa a quantidade de peças com lado y, e $\gamma$ representa a quantidade de peças com lado 1 que compõe o lado desse quadrado.

Por exemplo, o quadrado da figura 5 possui o comprimento do lado igual a $\mathrm{x}+\mathrm{y}+3$, pois um de seus lados é formado pela união dos lados de: uma peça rosa com medida $y$; uma peça laranja com medida $\mathrm{x}$ e três peças vermelhas com medidas iguais a 1. 
Figura 5: Quadrado de lado $x+y+3$

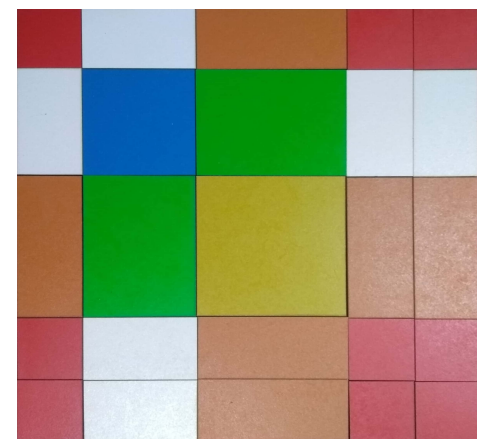

Note que a medida da área do quadrado da Figura 5 é $(x+y+3)^{2}$ e, usando-se as regras usuais da multiplicação, tem-se a seguinte relação:

$$
(x+y+3)^{2}=x^{2}+y^{2}+2 x y+6 x+6 y+9
$$

Observe que os coeficientes do polinômio do lado direito da Equação 1 indicam exatamente quantas peças de cada tipo foram utilizadas na construção do quadrado. É precisamente essa relação entre o quadrado (modelado algebricamente pelo lado esquerdo da equação 1) e os coeficientes da sua forma não fatorada (lado esquerdo da equação 1) que será usada para responder definitivamente à questão 1.

A Tabela 2 destaca a relação entre a quantidade de peças e os coeficientes do polinômio representado pela Figura 5.

Tabela 2: Relação entre os termos do polinômio 1 e a quantidade de peças.

\begin{tabular}{|c|c|}
\hline Termo do polinômio & Quantidade de peças \\
\hline $\mathrm{x}^{2}$ & uma peça amarela \\
\hline $\mathrm{y}^{2}$ & uma peça azul \\
\hline $2 \mathrm{xy}$ & duas peças verdes \\
\hline $6 \mathrm{x}$ & seis peças laranjas \\
\hline $6 \mathrm{y}$ & seis peças rosa \\
\hline 9 & nove peças vermelhas \\
\hline
\end{tabular}

A fim de tornar mais precisa a relação entre quadrados geométricos e polinômios, faremos uso da seguinte definição:

Definição 2 (Polinômio Quadrado Perfeito). Um polinômio de segundo grau nas variáveis x e y do tipo $\mathrm{Ax}^{2}+\mathrm{By}^{2}+\mathrm{Cxy}+\mathrm{Dx}+\mathrm{Ey}+\mathrm{F}$ é um polinômio quadrado perfeito quando existe $\alpha, \beta$ e $\gamma$ tal que

$$
\mathrm{Ax}^{2}+\mathrm{By}^{2}+\mathrm{Cxy}+\mathrm{Dx}+\mathrm{Ey}+\mathrm{F}=(\alpha \mathrm{x}+\beta \mathrm{y}+\gamma)^{2} .
$$

De acordo com a definição 2, um quadrado geométrico cujo lado mede $\alpha \mathrm{x}+\beta \mathrm{y}+\gamma$ está associado a um polinômio quadrado perfeito de forma que, para contar quantos quadrados é possível construir usando as peças do Algeplan, pode-se contar quantos polinômios quadrados perfeitos consegue-se construir levando em conta que, no caso do Algeplan, os coeficientes $\alpha, \beta$ e $\gamma$ são todos inteiros não negativos e estão sujeitos às restrições impostas pela quantidade limitada de peças. 
Para explicitar quais são as relações entre a quantidade de peças do Algeplan e os coeficientes $\alpha, \beta$ e $\gamma$, pode-se expandir o lado direito da equação 2 e estudar a seguinte igualdade:

$$
\mathrm{Ax}^{2}+\mathrm{By}^{2}+\mathrm{Cxy}+\mathrm{Dx}+\mathrm{Ey}+\mathrm{F}=\alpha^{2} \mathrm{x}^{2}+\beta^{2} \mathrm{y}^{2}+2 \beta \alpha \mathrm{xy}+2 \alpha \gamma \mathrm{x}+2 \beta \gamma \mathrm{y}+\gamma^{2}
$$

A equação 3 evidencia que os coeficientes A, B, C, D, E e F de um polinômio quadrado perfeito devem satisfazer as seguintes equações:

$$
\begin{aligned}
& \mathrm{A}=\alpha^{2} \\
& \mathrm{~B}=\beta^{2}
\end{aligned}
$$

$$
\begin{aligned}
& \mathrm{C}=2 \beta \alpha \\
& \mathrm{D}=2 \alpha \gamma
\end{aligned}
$$

$$
\begin{gathered}
\mathrm{E}=2 \beta \gamma \\
\mathrm{F}=\gamma^{2}
\end{gathered}
$$

para $\alpha, \beta$ e $\gamma$ convenientes.

Por exemplo, o polinômio $4 \mathrm{x}^{2}+\mathrm{xy}+1$ possui $\mathrm{A}=4, \mathrm{~B}=0, \mathrm{C}=1, \mathrm{D}=0, \mathrm{E}=0$ e $\mathrm{F}=1$ e não é um quadrado perfeito pois as equações 4,5 e 6 são incompatíveis. Já o polinômio $x^{2}+y^{2}+2 x y+6 x+6 y+9$ possui $\mathrm{A}=1, \mathrm{~B}=1, \mathrm{C}=2, \mathrm{D}=6, \mathrm{E}=6 \mathrm{e} \mathrm{F}=9$ e é um quadrado perfeito já que com $\alpha=1, \beta=1 \mathrm{e}$ $\gamma=3$ as igualdades $4,5,6,7,8,9$ são todas verdadeiras.

\section{Solução da questão 1}

Para se descobrir qual é o número máximo de quadrados que se pode formar com as peças, faremos uso da Tabela 3, na qual a primeira coluna contém os coeficientes dos termos de um polinômio quadrado perfeito genérico; na segunda coluna estão representadas as restrições que esses termos devem satisfazer de acordo com a quantidade de peças de cada tipo que compõe o Algeplan usado neste trabalho; na terceira e última coluna tem-se os valores (inteiros não negativos) de $\alpha, \beta$ e $\gamma$ que podem ser aplicados satisfazendo as restrições da segunda coluna.

Tabela 3: Valores de $\alpha, \beta$ e $\gamma$ - condição de existência

\begin{tabular}{|l|l|l|}
\hline $\begin{array}{l}\text { Coeficientes do } \\
\text { polinômio. }\end{array}$ & $\begin{array}{l}\text { Restrições devido a } \\
\text { quantidade de peças. }\end{array}$ & $\begin{array}{l}\text { Valores possíveis } \\
\text { para } \alpha, \beta \text { e } \gamma .\end{array}$ \\
\hline \hline$\alpha^{2}$ & $0 \leq \alpha^{2} \leq 4$ & $0 \leq \alpha \leq 2$ \\
\hline$\beta^{2}$ & $0 \leq \beta^{2} \leq 4$ & $0 \leq \beta \leq 2$ \\
\hline$\gamma^{2}$ & $0 \leq \gamma^{2} \leq 12$ & $0 \leq \gamma \leq 3$ \\
\hline $2 \beta \alpha$ & $0 \leq 2 \beta \alpha \leq 4$ & $0 \leq \beta \alpha \leq 2$ \\
\hline $2 \alpha \gamma$ & $0 \leq 2 \alpha \gamma \leq 8$ & $0 \leq \alpha \gamma \leq 4$ \\
\hline $2 \beta \gamma$ & $0 \leq 2 \beta \gamma \leq 8$ & $0 \leq \beta \gamma \leq 4$ \\
\hline
\end{tabular}

Analisando as três primeiras linhas da Tabela 3 pode-se observar que os valores de $\alpha, \beta$ e $\gamma$ ficam restritos a, no máximo, 3 possibilidades para $\alpha, 3$ para $\beta$ e 4 para $\gamma$. Sendo assim, as três primeiras linhas delimitam $3 \times 3 \times 4=36$ casos a serem considerados. No entanto, 8 desses casos não satisfazem as restrições explicitadas nas três últimas linhas da Tabela 3. Por exemplo, $\alpha=1, \beta=2$ e $\gamma=3$ não satisfaz a última linha da tabela já que $\beta \gamma=6>4$, o que significa que faltariam peças para construir um quadrado com lado igual ax $+2 \mathrm{y}+3$. São portanto $36-8=28$ os quadrados possíveis de se construir com as peças da Tabela 1 .

A seguir, pode-se ver na Tabela 4, quais são os quadrados que podem ser construídos, bem como sua representação algébrica. 
Tabela 4: Tabela com todas possibilidades de quadrados construtíveis com as 40 peças do Algeplan

\begin{tabular}{|c|c|c|c|c|}
\hline$\alpha$ & $\beta$ & $\gamma$ & Expressão Algébrica & Imagem \\
\hline \multirow{12}{*}{0} & \multirow{4}{*}{0} & 0 & 0 & \\
\hline & & 1 & 1 & $6 a$ \\
\hline & & 2 & 4 & $6 \mathrm{~b}$ \\
\hline & & 3 & 9 & $6 c$ \\
\hline & \multirow{4}{*}{1} & 0 & $y^{2}$ & $7 \mathrm{a}$ \\
\hline & & 1 & $y^{2}+2 y+1$ & $7 b$ \\
\hline & & 2 & $y^{2}+4 y+4$ & $7 \mathrm{c}$ \\
\hline & & 3 & $y^{2}+6 y+9$ & $7 \mathrm{~d}$ \\
\hline & \multirow{4}{*}{2} & 0 & $4 y^{2}$ & $8 \mathrm{a}$ \\
\hline & & 1 & $4 y^{2}+4 y+1$ & $8 \mathrm{~b}$ \\
\hline & & 2 & $4 y^{2}+8 y+4$ & $8 \mathrm{c}$ \\
\hline & & 3 & \multicolumn{2}{|c|}{ Seriam necessárias 12 peças do tipo y. } \\
\hline \multirow{12}{*}{1} & \multirow{4}{*}{0} & 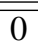 & $\overline{\mathrm{x}^{2}}$ & $9 \mathrm{a}$ \\
\hline & & 1 & $x^{2}+2 x+1$ & $9 \mathrm{~b}$ \\
\hline & & 2 & $x^{2}+4 x+4$ & $9 \mathrm{c}$ \\
\hline & & 3 & $x^{2}+6 x+9$ & $9 \mathrm{~d}$ \\
\hline & \multirow{4}{*}{1} & 0 & $x^{2}+2 x y+y^{2}$ & $10 \mathrm{a}$ \\
\hline & & 1 & $x^{2}+2 x y+y^{2}+2 x+2 y+1$ & $10 \mathrm{~b}$ \\
\hline & & 2 & $x^{2}+2 x y+y^{2}+4 x+4 y+4$ & $10 \mathrm{c}$ \\
\hline & & 3 & $x^{2}+2 x y+y^{2}+6 x+6 y+9$ & $10 \mathrm{~d}$ \\
\hline & \multirow{4}{*}{2} & 0 & $x^{2}+4 x y+4 y^{2}$ & $11 \mathrm{a}$ \\
\hline & & 1 & $x^{2}+4 x y+4 y^{2}+2 x+4 y+1$ & $11 \mathrm{~b}$ \\
\hline & & 2 & $x^{2}+4 x y+4 y^{2}+4 x+8 y+4$ & $11 \mathrm{c}$ \\
\hline & & 3 & Seriam necessári & tipo y. \\
\hline \multirow{12}{*}{2} & \multirow{4}{*}{0} & $\overline{0}$ & $4 \mathrm{x}^{2}$ & $12 \mathrm{a}$ \\
\hline & & 1 & $4 x^{2}+4 x+1$ & $12 \mathrm{~b}$ \\
\hline & & 2 & $4 x^{2}+8 x+4$ & $12 \mathrm{c}$ \\
\hline & & 3 & Seriam necessári & tipo $\mathrm{x}$. \\
\hline & \multirow{4}{*}{1} & 0 & $4 x^{2}+4 x y+y^{2}$ & $13 \mathrm{a}$ \\
\hline & & 1 & $4 x^{2}+4 x y+y^{2}+4 x+2 y+1$ & $13 b$ \\
\hline & & 2 & $4 x^{2}+4 x y+y^{2}+8 x+4 y+4$ & $13 \mathrm{c}$ \\
\hline & & 3 & \multicolumn{2}{|c|}{ Seriam necessárias 12 peças do tipo $\mathrm{x}$. } \\
\hline & \multirow{4}{*}{2} & 0 & \multicolumn{2}{|c|}{ Seriam necessárias 8 peças do tipo xy. } \\
\hline & & 1 & \multicolumn{2}{|c|}{ Seriam necessárias 8 peças do tipo xy. } \\
\hline & & 2 & \multirow{2}{*}{\multicolumn{2}{|c|}{$\begin{array}{l}\text { Seriam necessárias } 8 \text { peças do tipo xy. } \\
\text { Scárias } 12 \text { pecas do } 12 \text { do tino y }\end{array}$}} \\
\hline & & 3 & & \\
\hline
\end{tabular}


Figura 6: Quadrados perfeitos $\operatorname{com} \alpha=0 ; \beta=0$

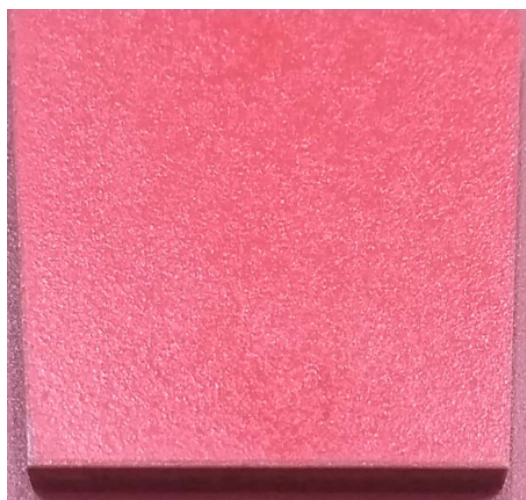

(a) $\alpha=0 ; \beta=0 ; \gamma=1$

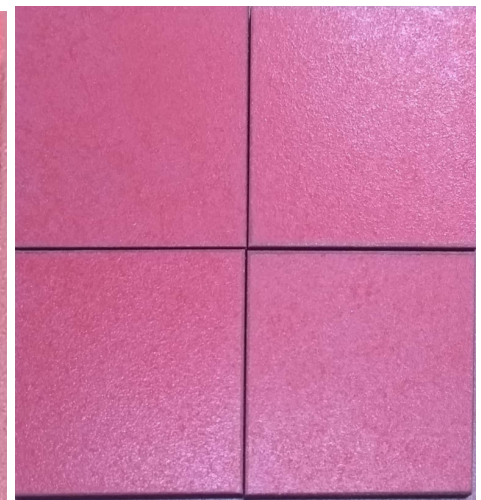

(b) $\alpha=0 ; \beta=0 ; \gamma=2$

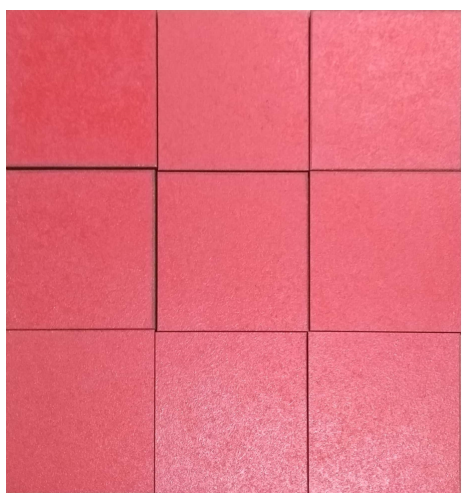

(c) $\alpha=0 ; \beta=0 ; \gamma=3$

Figura 7: Quadrados perfeitos $\operatorname{com} \alpha=0 ; \beta=1$

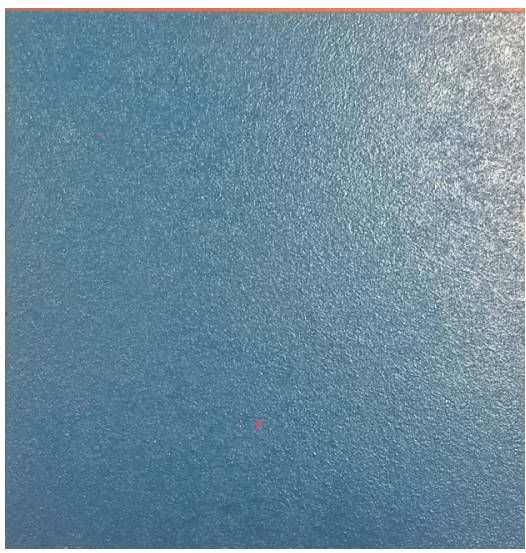

(a) $\alpha=0 ; \beta=1 ; \gamma=0$

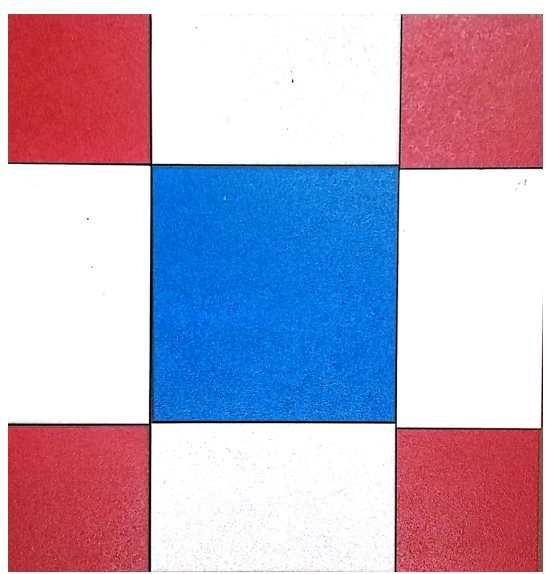

(c) $\alpha=0 ; \beta=1 ; \gamma=2$

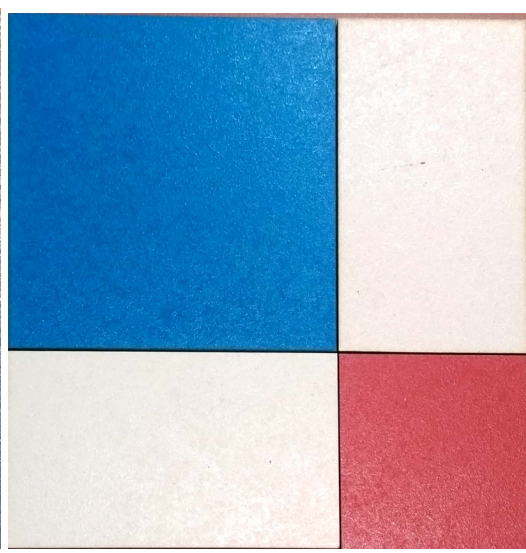

(b) $\alpha=0 ; \beta=1 ; \gamma=1$

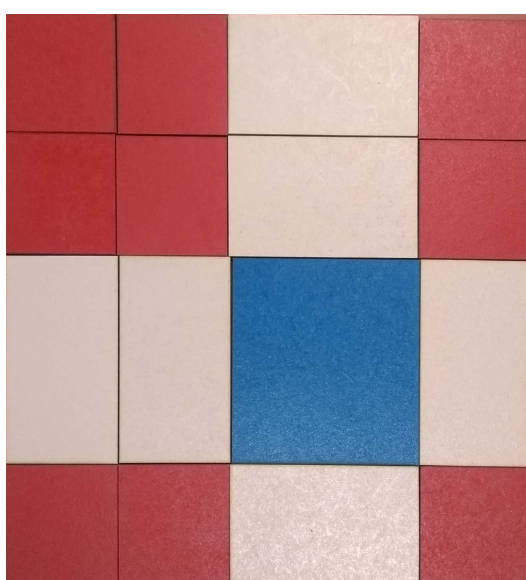

(d) $\alpha=0 ; \beta=1 ; \gamma=3$ 
Figura 8: Quadrados perfeitos $\operatorname{com} \alpha=0 ; \beta=2$

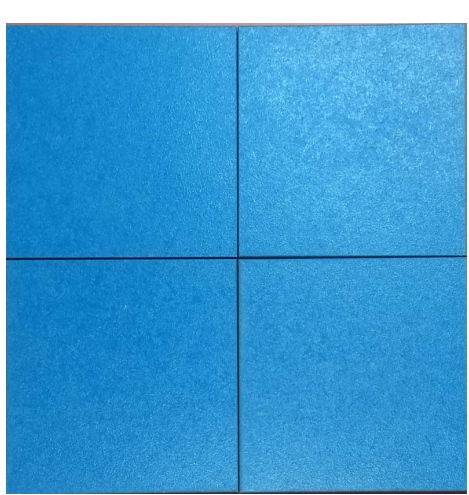

(a) $\alpha=0 ; \beta=2 ; \gamma=0$

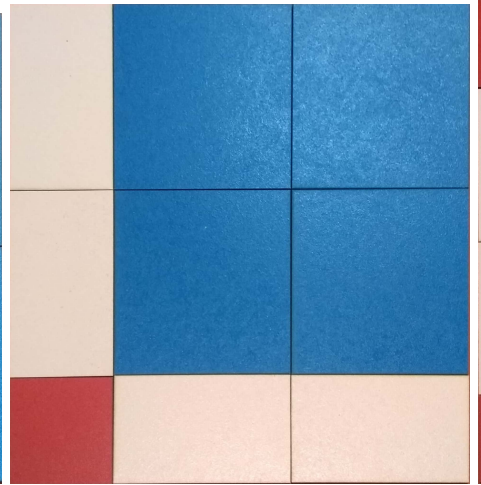

(b) $\alpha=0 ; \beta=2 ; \gamma=1$

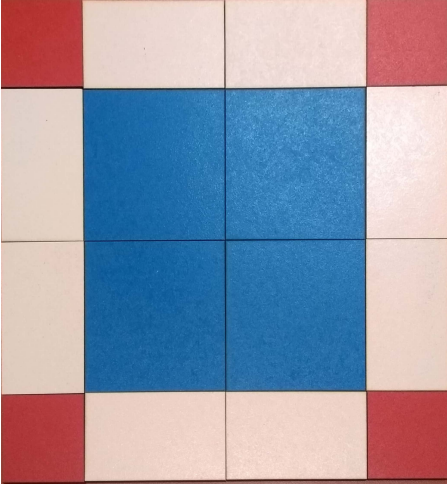

(c) $\alpha=0 ; \beta=2 ; \gamma=2$

Figura 9: Quadrados perfeitos $\operatorname{com} \alpha=1 ; \beta=0$

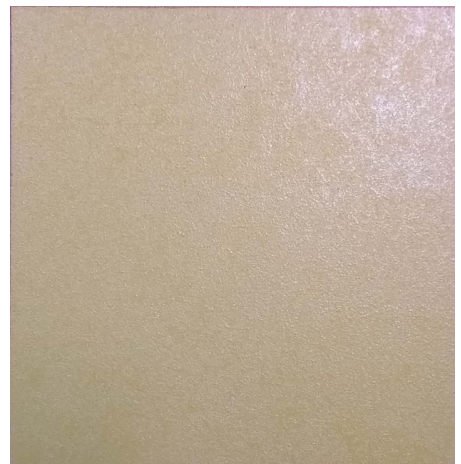

(a) $\alpha=1 ; \beta=0 ; \gamma=0$

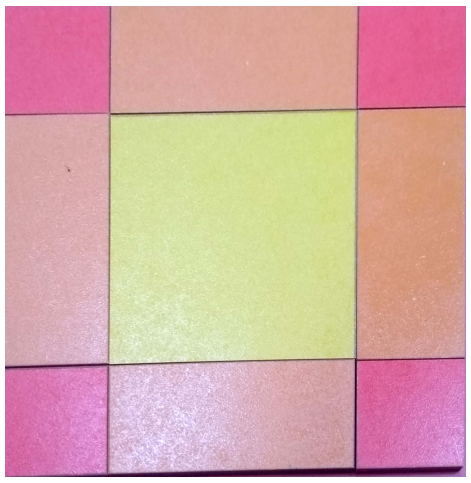

(c) $\alpha=1 ; \beta=0 ; \gamma=2$

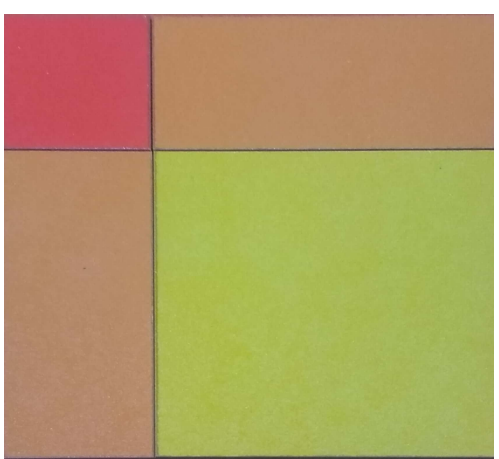

(b) $\alpha=1 ; \beta=0 ; \gamma=1$

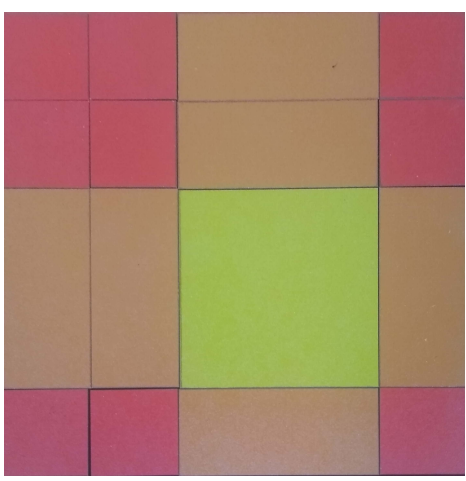

(d) $\alpha=1 ; \beta=0 ; \gamma=3$ 
Figura 10: Quadrados perfeitos $\operatorname{com} \alpha=1 ; \beta=1$

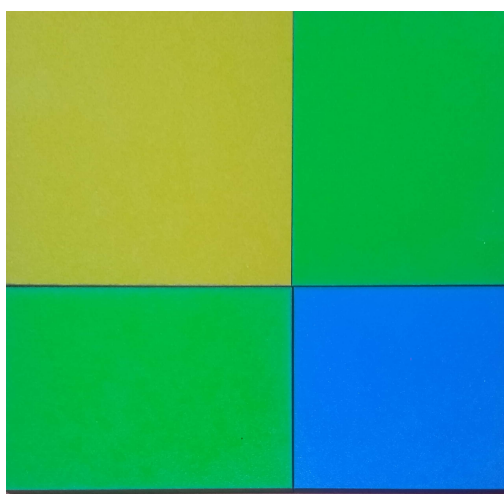

(a) $\alpha=1 ; \beta=1 ; \gamma=0$

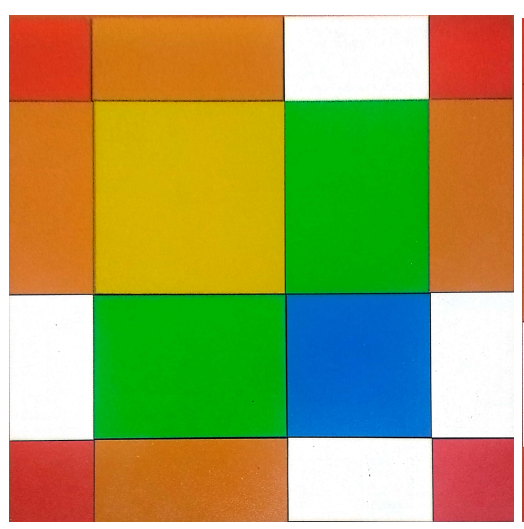

(c) $\alpha=1 ; \beta=1 ; \gamma=2$

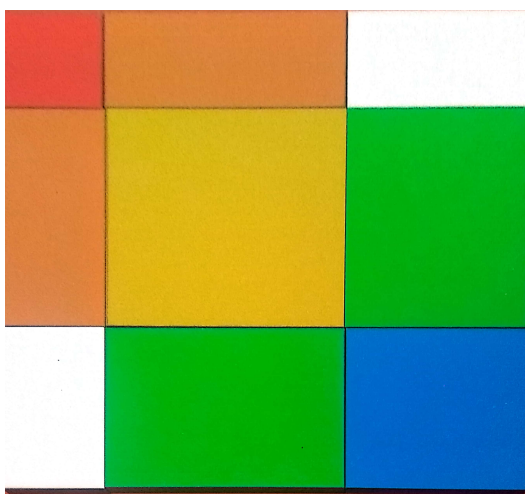

(b) $\alpha=1 ; \beta=1 ; \gamma=1$

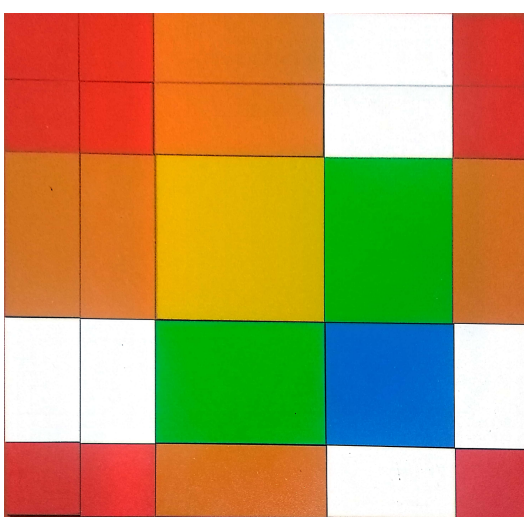

(d) $\alpha=1 ; \beta=1 ; \gamma=3$

Figura 11: Quadrados perfeitos $\operatorname{com} \alpha=1 ; \beta=2$

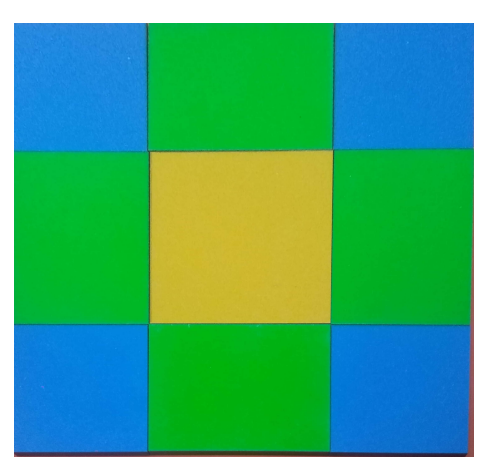

(a) $\alpha=1 ; \beta=2 ; \gamma=0$

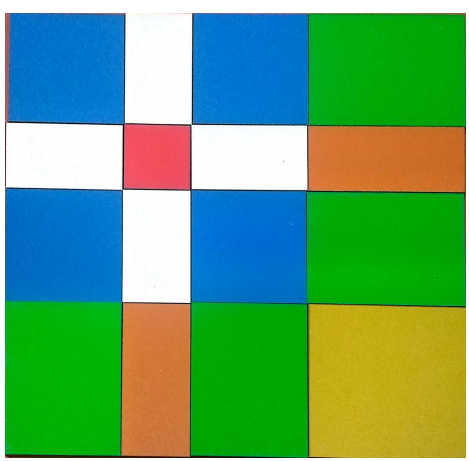

(b) $\alpha=1 ; \beta=2 ; \gamma=1$

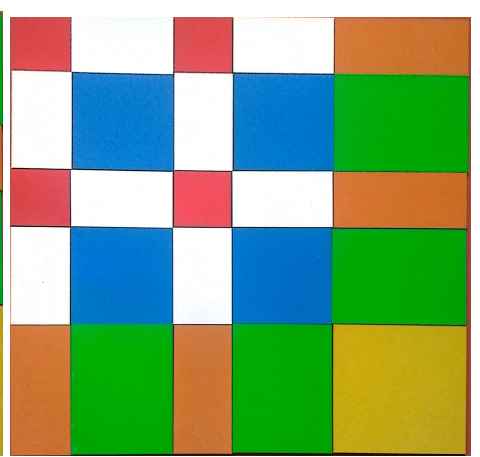

(c) $\alpha=1 ; \beta=2 ; \gamma=2$ 
Figura 12: Quadrados perfeitos $\operatorname{com} \alpha=2 ; \beta=0$

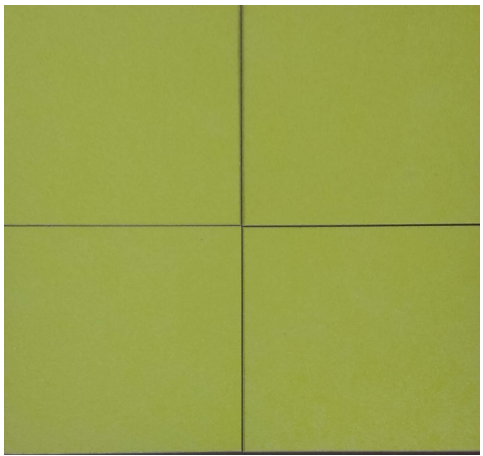

(a) $\alpha=2 ; \beta=0 ; \gamma=0$

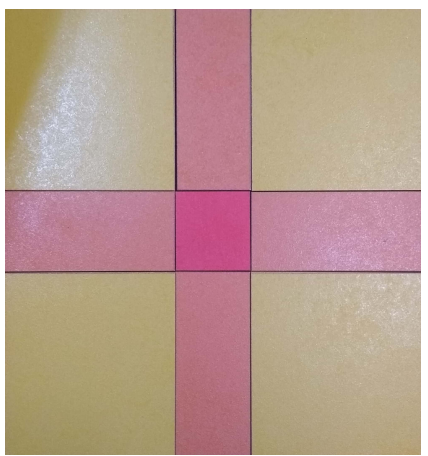

(b) $\alpha=2 ; \beta=0 ; \gamma=1$

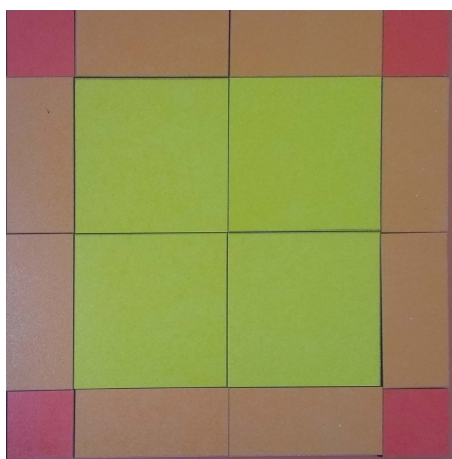

(c) $\alpha=2 ; \beta=0 ; \gamma=2$

Figura 13: Quadrados perfeitos $\operatorname{com} \alpha=2 ; \beta=1$

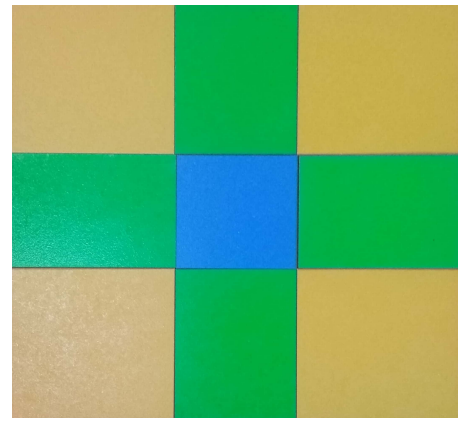

(a) $\alpha=2 ; \beta=1 ; \gamma=0$

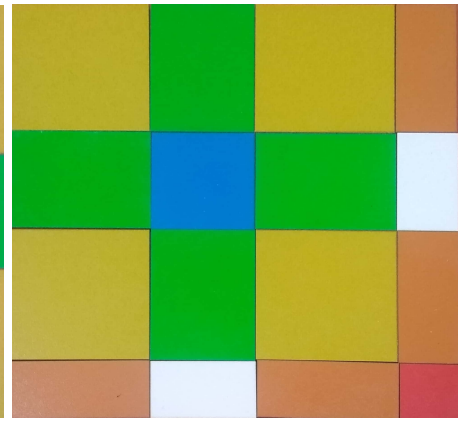

(b) $\alpha=2 ; \beta=1 ; \gamma=1$

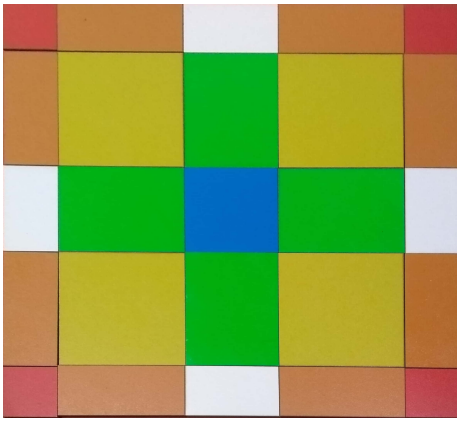

(c) $\alpha=2 ; \beta=1 ; \gamma=2$

\section{Sugestões para uso em sala de aula}

A fim de auxiliar o professor que deseja utilizar esta atividade em suas aulas, sugerimos alguns procedimentos baseados na nossa experiência com o uso deste material.

Quando se propõe a questão "quantos quadrados podem-se formar com a justaposição das peças do Algeplan" os alunos, na sua maioria, põem-se a trabalhar montando os quadrados usando, inicialmente, o método de tentativa e erro.

Apenas depois de certo tempo manipulando as peças é que algumas ideias de sistematização da contagem começam a surgir, mas ainda sem envolver a modelagem algébrica do problema.

Sendo assim, sugerimos que no início da atividade, logo após a apresentação e descrição do Algeplan, sejam fixadas as nomenclaturas $\mathrm{x}$, y e 1 para os lados das peças e também a relação de cada tipo de peça com sua representação algébrica, conforme a Tabela 1. Recomendamos que alguns exemplos sejam expostos na lousa, enfatizando a relação entre os coeficientes do polinômio de grau 2 com a quantidade de peças utilizadas na justaposição e, no caso específico de a justaposição resultar em um quadrado, a relação da quantidade de peças com os coeficientes da forma fatorada $(\alpha \mathrm{x}+\beta \mathrm{y}+\gamma)^{2}$ do polinômio. 
Tabela 5: Tabela para os alunos preencherem durante a aplicação

\begin{tabular}{|c|l|l|l|l|l|l|l|}
\hline & AMARELO & VERDE & AZUL & VERMELHO & ROSA & LARANJA & $\begin{array}{l}\text { EXPRESS ÃO } \\
\text { ALGÉBRICA }\end{array}$ \\
\hline 1 & & & & & & & \\
\hline 2 & & & & & & & \\
\hline$\ddots$ & & & & & & & \\
\hline
\end{tabular}

Uma maneira de chamar a atenção do aluno para a relação entre a quantidade de peças usadas em um quadrado e a expressão algébrica correspondente é solicitar que, em uma tabela similar à Tabela 5, a cada quadrado encontrado, seja anotada a quantidade de peças de cada cor que foram utilizadas bem como a expressão algébrica a ele relacionada. Dessa forma, mesmo que algum estudante não descubra por si só um argumento que justifique que a quantidade exata de quadrados é 28 , ficará mais fácil a compreensão da explicação dada posteriormente pelo professor.

No entanto, a maneira de desenvolver a atividade dependerá do nível de maturidade dos alunos envolvidos e do tempo disponível, cabendo ao professor mediar e conduzir a execução do trabalho.

\section{Considerações finais}

Vale notar que, ao se considerar uma quantidade de peças diferente da apresentada na Tabela 1, uma nova análise de possibilidades precisa ser feita, seguindo o raciocínio da Tabela 3, usando as novas restrições. Este fato abre a possibilidade do uso de tal atividade em mais de uma ocasião, modificando a quantidade de um ou mais tipos de peças do Algeplan.

Ainda: mesmo que o professor não tenha acesso a kits do material concreto, existem versões virtuais do Algeplan que podem ser usadas em computadores e smartfones, conforme [4], [5]. O uso da versão virtual pode ser uma outra alternativa de implementação desta atividade, inclusive com diferentes quantidades de peças.

Por fim, quando se observa a Figura 14, percebe-se que existe mais de uma maneira de rearranjar as peças do Algeplan para se construir um quadrado. Descobrir, para cada quadrado, quantas "maneiras diferentes" as peças podem ser rearranjadas, é também um outro problema de contagem que pode ser trabalhado com o auxílio deste material.

Figura 14: O quadrado de lado $2 y+2$ montado de duas maneiras diferentes
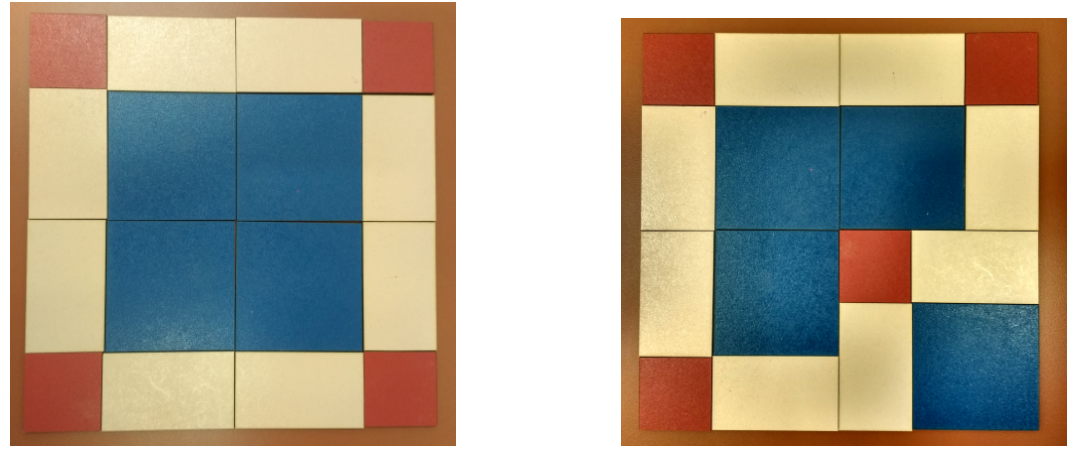


\section{Agradecimentos}

Agradecemos ao Laboratório de Ensino e Pesquisa em Educação Matemática - Furg-SAP pelo empréstimo do material usado neste trabalho, e as valiosas sugestões da Editora responsável pelo parecer.

\section{Referências}

[1] Ausubel, D. P. Aquisição e retenção de conhecimentos: uma perspectiva cognitiva. Lisboa: Plátano, 1. 2003.

[2] Fanti, E. L. C; Rosa, R. A., Dias, F. M.; Medeiros, L. T.; O Algeplan como um recurso didático na exploração de expressões algébricas e fatoração. In: III Bienal da SBM, 2006, Goiânia. Pôsteres da III Bienal da SBM, 2006.

[3] Poleto, C. S., Algeplan, Álgebra e Geometria : entendendo práticas matemáticas como jogos de linguagem. Trabalho de conclusão de curso - UFRS, Porto Alegre, 2010.

[4] Silva, P. L.; Basso, M. V. A. "Algeplan Virtual: Um Recurso para o Ensino de Operações com Polinômios. Anais do XI Encontro Nacional de Educação Matemática”- ISSN 2178-034X, 2013.

[5] Massante, K. A. S. C. C.; Barbosa, A. C. M.; Gonçalves, I. M. “Atividades Utilizando o Algeplan no Software Geogebra. XII Encontro Nacional de Educação Matemática”, 2016.

Karina Rodriguez de Fraga Universidade Federal do Rio Grande <fragak14@gmail.com>

Leandro Sebben Bellicanta Universidade Federal do Rio Grande <leandrobellicanta@furg.br>

Recebido: 29/10/2019

Publicado: 08/07/2020 